\title{
Nonlinear magnetic-coupled flutter-based aeroelastic energy harvester: Modeling, simulation and experimental verification
}

\author{
Kui Li, Zhichun Yang ${ }^{\text {a) }}$, Yingsong Gu ${ }^{\text {a) }}$, Shun He, Shengxi Zhou \\ School of Aeronautics, Northwestern Polytechnical University, Xi'an 710072, China
}

\begin{abstract}
Aeroelastic energy harvesting can be used to power wireless sensors embedded into bridges, ducts, high-altitude buildings, etc. One challenging issue is that the wind speed in some application environments is low, which leads to an inefficiency of aeroelastic energy harvesters. This paper presents a novel nonlinear magnetic-coupled flutter-based aeroelastic energy harvester to enhance energy harvesting at low wind speeds. The presented harvester mainly consists of a piezoelectric beam, a two-dimensional airfoil, two tip magnets and two external magnets. The function of magnets is to reduce the cut-in wind speed of the flutter-based aeroelastic energy harvester and enhance energy harvesting performance at low wind speeds. A theoretical model is deduced based on Hamilton's principle, theory of aeroelasticity, Kirchhoff's laws and experimental measurements, etc. A good agreement is found between numerical simulation and experimental results, which verifies the accuracy of the theoretical model. Stability analysis is provided to determine the characteristics of the presented harvester. More importantly, it is numerically and experimentally verified that the presented harvester has a much lower cut-in wind speed (about $1.0 \mathrm{~m} / \mathrm{s}$ ) and has a better energy harvesting performance at a low wind speed range from $1.0 \mathrm{~m} / \mathrm{s}$ to $2.9 \mathrm{~m} / \mathrm{s}$, when compared with traditional flutter-based aeroelastic energy harvesters.
\end{abstract}

Keywords: Aeroelastic energy harvesting, magnetic coupled, nonlinear modeling, broadband, enhance harvesting efficiency

\section{Introduction}

\footnotetext{
a ) Authors to whom correspondence should be addressed. E-mails: yangzc@nwpu.edu.cn (Z. Yang); guyingsong@nwpu.edu.cn (Y.Gu).
} 
Energy harvesting has been receiving more and more research interests in the past ten years. It is expected to solve the challenging issue of energy supply for wireless sensors and small portable devices by eliminating the dependency of chemical batteries via energy harvesting. There are plentiful ambient energy sources including mechanical vibrations, electromagnetic radiation, human motions, and wind flows, which can be harvested based on reasonable devices [1-6]. Many studies have been conducted on harvesting energy from ambient vibrations using piezoelectric materials [7-12]. However, piezoelectric energy harvesting from wind energy has received relatively little attention at the same time.

Aeroelastic energy harvesting methods and devices mainly include vortex-induced vibration (VIV) [1319], galloping [20-26], flutter [27-32] based energy harvesters. VIV based energy harvesters will efficiently work when the vortex shedding frequency becomes nearly equal to one of their natural frequencies. In 2012 , Akaydin et al. [13] experimentally obtained a peak output power of $0.1 \mathrm{~mW}$ at the wind speed of $1.192 \mathrm{~m} / \mathrm{s}$ using a VIV energy harvester. Goushcha et al. [14] explored the driving mechanisms of VIV energy harvesting by using the particle image velocimetry. Dai et al. [15] and Zhang et al. [16] made a complete analysis of different bluff bodies of VIV based energy harvesters for improving energy harvesting performance. Weinstein et al. [17] tested the energy harvesting performance of a piezoelectric beam in the real heating, ventilation and air conditioning flow. Zhang et al. [18] studied the fluid-induced vibration energy harvesting subject to different attack angles. Zhou and Wang [19] presented a dual serial vortex-induced vibration energy harvesting system for enhanced energy harvesting. Galloping occurs to a flexible base support with a bluff body [20, 21]. Ewere et al. [22] experimentally investigated galloping piezoelectric energy harvesters with square bluff bodies. Zhao et al. $[23,24]$ experimentally investigated the influence of bluff body shape on the energy harvesting performance of piezoelectric galloping energy harvesters. Abdelkefi et al. [25] deduced the distributed parametric model of cantilever beam galloping energy harvesters, and its efficiency was experimentally verified. Tan and Yan [26] presented the analytical solutions for the optimal design of galloping-based piezoelectric energy harvesters.

Flutter phenomenon occurs when the frequencies of torsion and bending modes coalesce with each other [27, 28]. Bryant and Garcia [29] originally proposed a flutter-based aeroelastic energy harvester, and they found that the harvester oscillated in a limit cycle at higher wind speeds above a critical wind speed. Aquino et al. [30] 
modeled a flutter-based aeroelastic energy harvester using Computational Fluid Dynamics (CFD) method and experimentally obtained a peak-to-peak output voltage of $8.72 \mathrm{~V}$ and a short-circuit current of $1 \mathrm{~mA}$ when subjected to the wind speed of $2.3 \mathrm{~m} / \mathrm{s}$. Wu et al. [31] designed and modeled a flutter-based aeroelastic energy harvester with double plunge degrees of freedom, and they pointed out that the first (windward) supporting device should be deployed close to the airfoil leading edge to reduce the cut-in speed.

Recently, Yan et al. [32] used the nonlinear energy sink as an effective strategy for passive flutter control. This research shows that the nonlinear stiffness could obviously change the dynamic characteristics of a flutter system. Recently, Zhao et al. [33] presented an impact-based broadband aeroelastic energy harvester based on nonlinear mechanism, and experimental results verified the improvement of energy harvesting performance of their presented harvester. Their research demonstrates that the dynamic characteristics and the energy harvesting performance of galloping-based energy harvesters could be changed and improved by adding nonlinear terms. Meanwhile, introducing nonlinearity by adding magnets to achieve nonlinear monostable [34], bistable [35, 36], and tristable [37] configurations was reported to improve energy harvesting performance from base vibration. Nasser and Dai et al. [38, 39] investigated the vortex-induced energy harvester with introducing nonlinear magnetic force to enhance energy harvesting efficiency. They analyzed how the distance of magnets affect upon the performance of harvesters. Huynh [40] also designed a bisatble VIV energy harvester to enhance the displacement of structure and improve the energy harvesting performance. Alhadidi et al. [41] demonstrated a bistable galloping energy harvester composed by magnets to broaden the response bandwidth under varying flow speeds. The concept with magnetic coupling has been introduced to enhance the wind-induced energy harvesting efficiency, however, the related research is still rare and it need structural breakthrough for traditional flutter-based energy harvester.

This paper presented a novel nonlinear magnetic-coupled flutter-based aeroelastic energy harvester (NMFAEH) to decrease the cut-in wind speed and enhance energy harvesting efficiency at the low wind speed range. Two mounted-in magnets and two external magnets are used to produce the nonlinear magnetic force, which could change the equivalent stiffness of the harvester and lead to a much lower critical flutter speed. A theoretical model of the NMFAEH is deduced based on Hamilton's principle, theory of aeroelasticity, and experimental measurements. Stability analysis is performed to determine the characteristics of the NMFAEH. 
Numerical simulation and wind-tunnel experiments is performed to verify the design and theoretical analysis.

\section{Theoretical modeling}

\subsection{Modeling of the NMFAEH}

The structural schematic of the presented NMFAEH is shown in Figure 1. It consists of a piezoelectric beam with two tip magnets, a rigid airfoil with a hinged connection to the free end of the beam, and two external magnets are installed in the front of the leading edge of the airfoil. Inspired from the investigation in which the nonlinear magnetic force is introduced for enhanced broadband energy harvesting from base vibrations [34, 36, 42]. The angular orientation and distance of magnets can be changed to alter the nonlinear magnetic force to adjust the equivalent stiffness of the harvester. In this paper, different from existing flutter-based aeroelastic energy harvesters (FAEH), the nonlinear magnetic force produced by the interaction among two tip magnets and two external magnets is used to tune the dynamic characteristics of the harvester and change the critical flutter speed consequently. The two external magnets are symmetrically installed which is shown in Figure 1. The distance $l$ between external magnets and tip magnets is initially fixed as $20 \mathrm{~mm}$ to insure the airfoil not to touch with the external magnets under its plunging movement. Based on the priori knowledge, for fixed orientation of the magnets, the parameter value of $d=124 \mathrm{~mm}$ is theoretically estimated by using dipoledipole magnetic model $[34,36]$ and fine adjusted manually to produce the suitable softening nonlinear magnetic force in the followings. The corresponding aeroelastic equation of the motion coupled with the nonlinear magnetic force is developed to study the feasibility of the presented method.

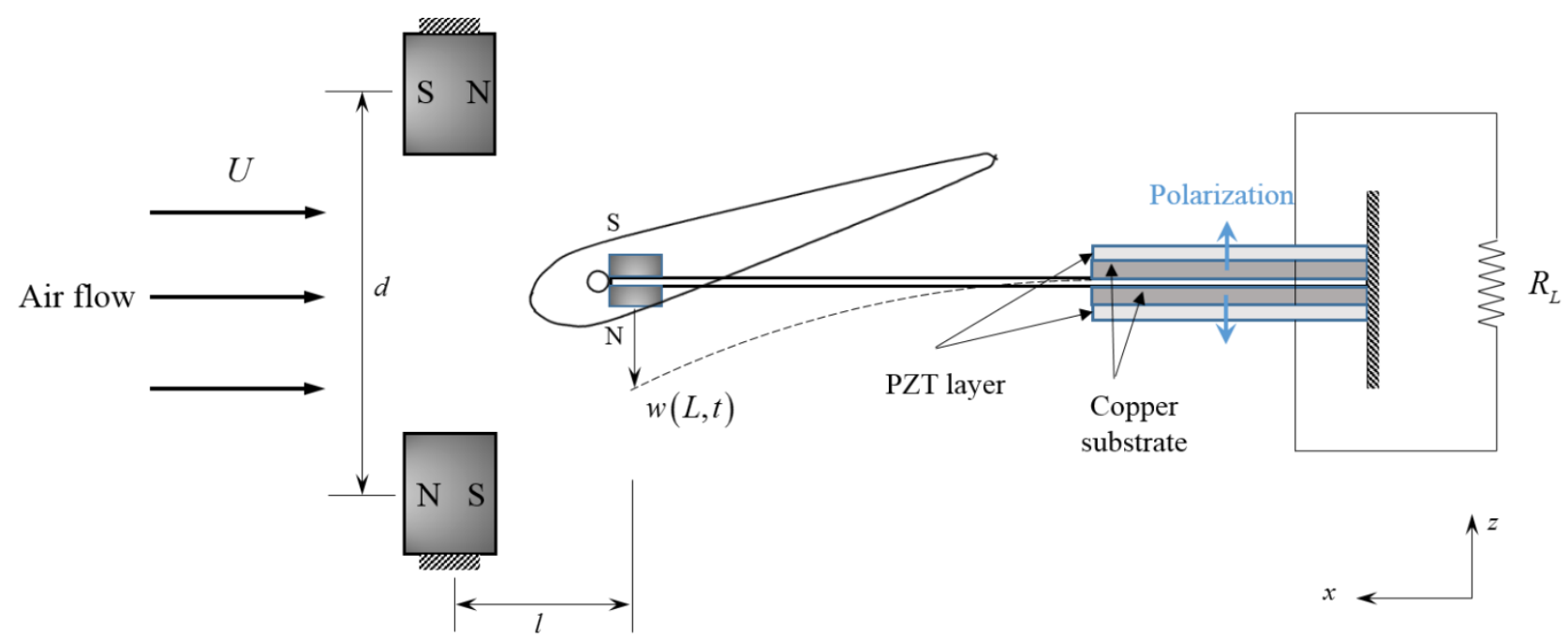

Figure 1. Structural schematic of the presented NMFAEH. 


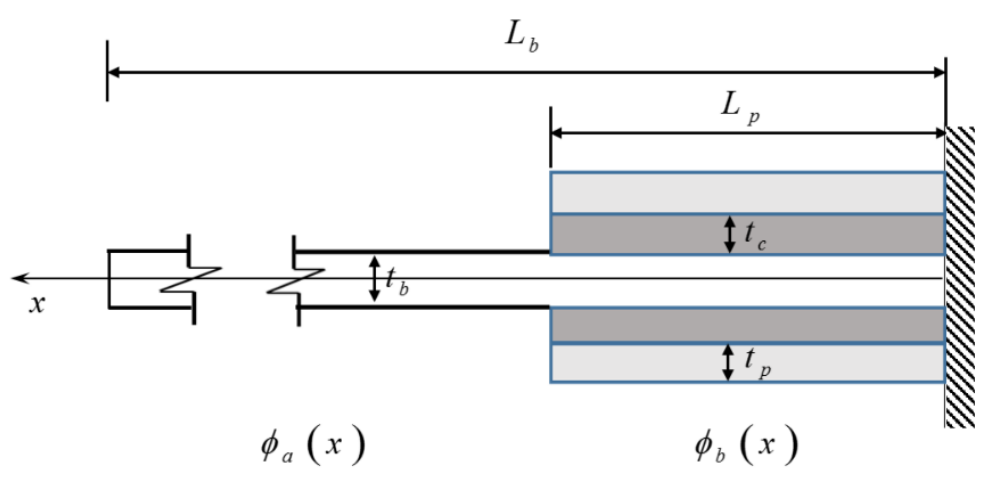

Figure 2. Cross-section view of the piezoelectric beam.

In experiments, the piezoelectric patches and their copper substrates are glued on the cantilever beam at the clamped end, as shown in Figure 2. With the full consideration of the geometric nonlinearity induced by the large deformation of the beam, the nonlinear magnetic force, the aerodynamic load on the two-dimensional airfoil, and the external load resistance, a theoretical model is deduced based on Hamilton's principle, theory of aeroelasticity, Kirchhoff's laws and experimental measurements. By introducing the geometric nonlinear condition [43] formulated as $S_{1}=-z w^{\prime \prime}\left[1+\frac{1}{2} w^{\prime 2}\right]$, the kinetic energy and the potential energy of the harvester can be described as:

$$
\begin{gathered}
T^{*}=\frac{1}{2} \int_{0}^{L_{b}} \int_{A_{b}} \rho_{b} \dot{w}^{2} d A d x+\frac{1}{2} \int_{0}^{L_{p}} \int_{A_{c}} \rho_{c} \dot{w}^{2} d A d x+\frac{1}{2} \int_{0}^{L_{p}} \int_{A_{p}} \rho_{p} \dot{w}^{2} d A d x+\frac{1}{2} m_{T} \dot{w}^{2} \delta(x-L) \\
+\frac{1}{2} m_{F} \dot{w}^{2} \delta(x-L)+m_{F} x_{\alpha} \dot{\alpha} \dot{w} \delta(x-L)+\frac{1}{2} I_{p} \dot{\alpha}^{2} \\
U^{*}=\frac{1}{2} \int_{0}^{L_{b}} \int_{A_{b}} E_{b} S_{1}^{2} d A d x+\frac{1}{2} \int_{0}^{L_{p}} \int_{A_{c}} E_{c} S_{1}^{2} d A d x+\frac{1}{2} \int_{0}^{L_{p}} \int_{A_{p}} c_{11}^{E} S_{1}^{2} d A d x \\
-\frac{1}{2} \int_{0}^{L_{p}} \int_{A_{p}} \varepsilon_{33}^{S} \frac{V^{2}}{t_{p}^{2}} d A d x-\int_{0}^{L_{p}} \int_{A_{p}} S_{1} e_{31} \frac{V}{t_{p}} d A d x
\end{gathered}
$$

where $w(x, t)$ is the transverse deflection of the harvester and $S_{1}$ is the axial strain; $\alpha(t)$ is the pitching displacement of the airfoil; $V(t)$ is the output voltage of the harvester. The symbols $\rho, E$ and $A$ are the material density and the Young's modulus and the cross-section the beam, respectively. The subscript $b, c$ and $p$ represent to the middle beam, copper substrate and piezoelectric patch, respectively. $c_{11}^{E}$ is the modulus of piezoelectric patch for short-circuit electrodes. $e_{31}$ and $\varepsilon_{33}^{S}$ are the piezoelectric constant and dielectric 
constant under the constant strain condition, respectively. $t_{p}$ is the thickness of the piezoelectric patch. $m_{F}$ is the mass of the airfoil. $\quad m_{T}$ is the total mass including two tip magnets and the connection components. $x_{\alpha}$ is the static unbalance parameter which is the non-dimensional distance between the center of gravity of airfoil and the axis of rotation. $I_{p}$ and $b$ are the mass moment of inertia and the semi chord length of the airfoil, respectively. $\delta(x)$ is the Dirac function of the variable $x$.

The work of the non-conservative external forces and the applied circuit is:

$$
W_{n c}^{*}=Q_{L} w \delta(x-L)+Q_{M} \alpha+F_{m} w \delta(x-L)+Q V
$$

where $Q_{L}$ and $Q_{M}$ are the aerodynamic lift and its moment applied on the airfoil, respectively. $F_{m}$ is the nonlinear magnetic force acting on the tip of beam. $Q$ is the total electric charge on the electrodes of the piezoelectric transducers.

Based on the normalized mode shapes as shown in Appendix A, the modal truncation method is introduced to consider the dominant first bending mode, as follows:

$$
w(x, t)=\phi_{1}(x) h(t)
$$

where the subscript 1 stands for the first normalized bending mode. $h(t)$ is the corresponding modal

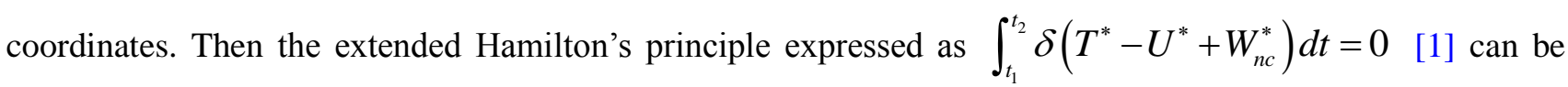
applied to derive the magneto-electro-aeroelastic governing equations of the NMFAEH. The final governing equations are obtained, as follows:

$$
\begin{gathered}
M \ddot{h}+m_{F} x_{a} b \ddot{\alpha}+C_{h} \dot{h}+K_{1} h+K_{3} h^{3}+\theta V=-Q_{L}+F_{m} \\
m_{F} x_{a} b \ddot{h}+I_{p} \ddot{\alpha}+K_{\alpha} \alpha=Q_{M} \\
C_{p} \dot{V}+\frac{V}{R}-\theta \dot{h}=0
\end{gathered}
$$

where $M$ is the total equivalent mass of the piezoelectric beam. $K_{1}$ and $K_{3}$ are the equivalent linear and nonlinear coefficients of the stiffness, respectively. $\theta=z_{m} e_{31} w_{p} \phi_{1}^{\prime}\left(L_{p}\right)$ is the electromechanical coupling coefficient. The equivalent capacitance of the piezoelectric patches for series connection in this study is 
$C_{p}=\frac{\varepsilon_{33}^{S} w_{p} L_{p}}{2 h_{p}}$, where $w_{p}$ is the width of the piezoelectric patch and $L_{p}$ is the length of the piezoelectric patch. $K_{\alpha}$ is the pitching stiffness of the airfoil (it is set to zero in this paper, because there is no torsional spring in Figure 1). The damping coefficient $C_{h}$ is introduced as the first order plunging damping. The airfoil is hinged with lubricated bearing, therefore, we didn't add the pitching damping to the model. $R$ is the load resistance.

The output power RMS is defined as:

$$
P=\frac{\left(V_{m} / \sqrt{2}\right)^{2}}{R}
$$

where $V_{m}$ is the amplitude of the output voltage.

\subsection{Identification of the nonlinear magnetic force}

The magnetic field is very sensitive to the relative location of the interacting magnets. Therefore, it is very hard to theoretically calculate the exact nonlinear magnetic force precisely with in the large deformation. The theoretical method in Ref. [34] and the experimental measurement in Ref. [37] were widely used. This paper uses a different experimental measurement method to identify the nonlinear magnetic force. The experimental setup is exhibited in Figure 3. In detail, a series of different weights are used to exert the horizontal pulling force at the tip of the beam. By utilizing a laser displacement sensor, the tip displacement of the beam can be measured simultaneously. So the stiffness of the beam without the magnetic force is firstly measured. Then with the external magnets coupling, the beam maintains the static equilibrium under the interaction of the pulling force, the elastic restoring force and the external nonlinear magnetic force. Therefore, the relationship between the external nonlinear magnetic force $F_{m}$ and the tip displacement $h$ is experimentally identified. 


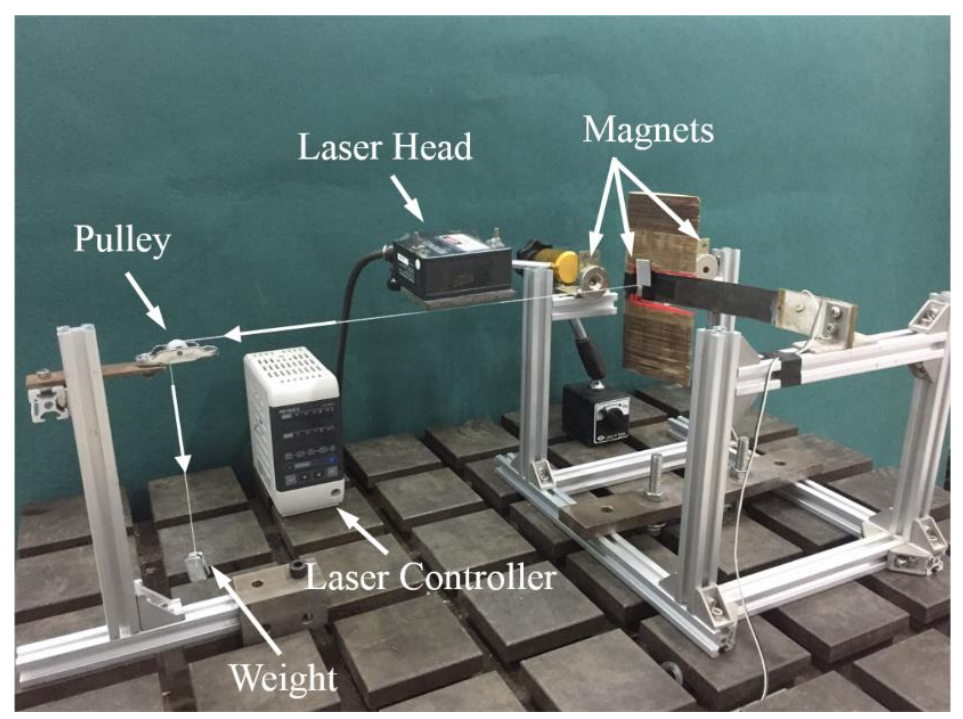

Figure 3. Experimental set-up for the identification of the nonlinear magnetic force.

The experimentally measured data and numerically fitted curves are shown in Figure 4. One should recall that the magnetic force is on the right side of Eq. (5) as an external forcing term. So the equivalent nonlinear restoring force which includes the contribution of the nonlinear magnetic force $-F_{m}$ and the elastic restoring force of the clamped piezo-beam is shown in Figure 4(a). Therefore, the magnetic force is calculated by subtracting the elastic restoring force from the equivalent nonlinear restoring force. The constitution between measured magnetic force and beam tip displacement is shown in Figure 4(b). Consequently, a polynomial expression is used to fit the nonlinear magnetic force, as follows:

$$
F_{m}=K_{m a g 1} h+K_{m a g 3} h^{3}
$$

where $K_{m a g 1}=10.2 \mathrm{~N} / \mathrm{m}$ and $K_{m a g 3}=-1.2 \times 10^{4} \mathrm{~N} / \mathrm{m}^{3}$ are identified in experiments and used in simulations later.

It is found in Figure 4(b) that the magnetic force is attractive in the tip displacement range of [-0.03 m, 0.03 $\mathrm{m}]$. Therefore, the harvester is softened by the magnetic field in this range. However, the magnetic field will harden the harvester when the tip displacement is outside the above range. It can be concluded that the nonlinear magnetic force could regulate the stiffness of the harvester while keeping it monostable. 
(a)

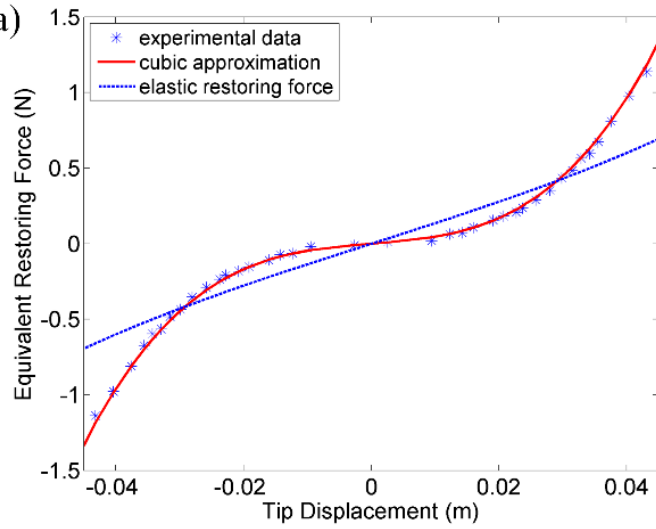

(b)

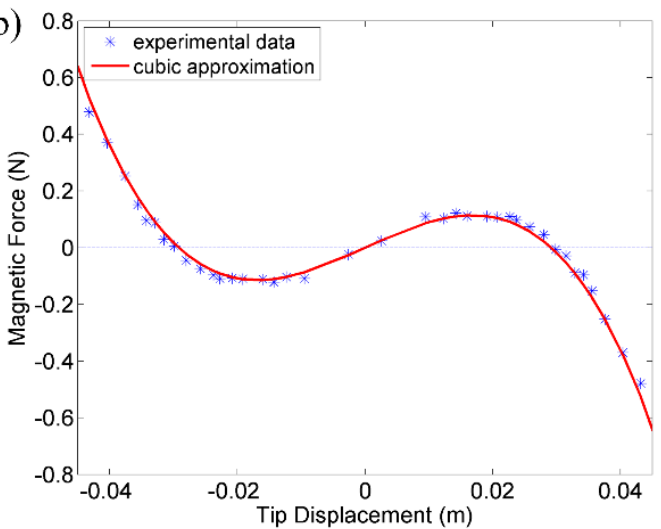

Figure 4. (a) The equivalent nonlinear restoring force; (b) the nonlinear magnetic force .

Figure 5 shows the potential energy and the equivalent stiffness of the NMFAEH and the FAEH. It is found in Figure 5(a) that the potential well of the NMFAEH is much flatter than that of the FAEH around the equilibrium position. This means that the NMFAEH can move to the farther distance near its equilibrium position when increasing the same value of potential energy. In other words, the NMFAEH may provide a larger deformation which will benefit the energy harvesting under a constant wind speed. The stiffness of the NMFAEH is softened by the attractive nonlinear magnetic force which is illustrated in Figure 5(b). Since the critical flutter speed of the FAEH is principally dominated by the structural stiffness, the softened effect could reduce the critical flutter wind speed of the NMFAEH. Therefore, the NMFAEH is more suitable to harvest energy in the lower wind speed range than the FAEH. As the increase of the tip displacement, the shape of the potential energy function of the NMFAEH becomes much steeper than that of the FAEH. This indicates that the stiffness of the NMFAEH is hardened by the repulsive nonlinear magnetic force in this case and protect the harvester against divergence. From the physical point of view, the NMFAEH will be easier to enter into limit cycle oscillations and have a better energy harvesting performance at the low wind speed range. 
(a)

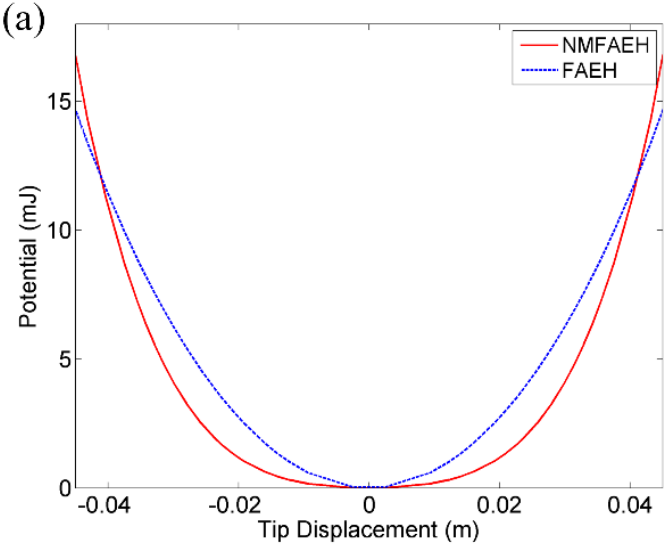

(b)

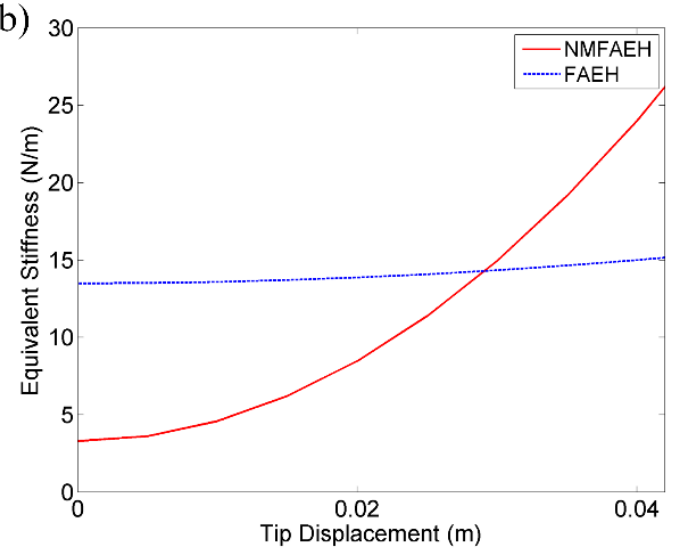

Figure 5. (a) Potential energy of NMFAEH and FAEH; (b) the equivalent nonlinear stiffness.

\subsection{Stall aerodynamics model}

In the presented model, there is no torsion spring acting on the rotation axis of the airfoil. The airfoil is prone to the experience large angle of attack during its movement under the flutter condition, which will cause the aerodynamic stall phenomenon [44]. For the quasi-steady stall model, the aerodynamic force has been evaluated using the cubic polynomial approximation or the piecewise linear function as mentioned in Refs. [25, $29,45,46]$. In this work, the piecewise linear function is employed to model the aerodynamic force considering the stall effect, as follows:

$$
\left\{\begin{array}{c}
Q_{L}=\rho V^{2} b s C_{L}\left(\alpha_{e f f}\right) \\
Q_{M}=\rho V^{2} b^{2} s\left(\frac{1}{2}+a\right) C_{L}\left(\alpha_{e f f}\right)-\frac{1}{2} \pi \rho V b^{3} s \dot{\alpha}
\end{array}\right.
$$

where $C_{L}\left(\alpha_{e f f}\right)$ is the quasi-steady aerodynamic lift coefficient. $\alpha_{\text {eff }}$ is the effective angle of attack due to the instantaneous motion of the airfoil and it is given by [29]:

$$
\alpha_{e f f}=\alpha+\frac{\dot{h}}{U}+\left(\frac{1}{2}-a\right) b \frac{\dot{\alpha}}{U}
$$

The piecewise linear function of the aerodynamic stall lift coefficient can be expressed as:

$$
\begin{aligned}
& C_{L}\left(\alpha_{\text {eff }}\right)=a_{o L} \alpha_{e f f}-\Delta C_{L}\left(\alpha_{\text {eff }}\right) \\
& \frac{\partial \Delta C_{L}}{\partial \alpha_{\text {eff }}}= \begin{cases}0, & -\bar{\alpha}_{1} \leq \alpha_{\text {eff }} \leq \bar{\alpha}_{1} \\
6.3228, & \bar{\alpha}_{1} \leq \alpha_{\text {eff }} \leq \bar{\alpha}_{2},-\bar{\alpha}_{2} \leq \alpha_{\text {eff }} \leq-\bar{\alpha}_{1} \\
5.9, & \bar{\alpha}_{2} \leq \alpha_{\text {eff }}, \alpha_{\text {eff }} \leq-\bar{\alpha}_{2}\end{cases}
\end{aligned}
$$


where $\alpha_{0 L}$ is the linear lift coefficient. $\Delta C_{L}\left(\alpha_{\text {eff }}\right)$ is the difference between linear and nonlinear components of the aerodynamic coefficient curve.

\section{Stability analysis and numerical simulation}

In order to analyze the influence of the nonlinear magnetic force, the cut-in wind speeds of the NMFAEH and FAEH are determined by stability analysis in this section. The energy harvesting capacity is also compared between NMFAEH and FAEH by time domain simulation.

\subsection{Stability analysis}

Flutter is a kind of self-excited vibrations. The critical flutter wind speed (it can also be called cut-in wind speed of energy harvesting) is an important parameter of the NMFAEH, which decides the lowest working wind speed. The cut-in wind speed mainly depends on linear aerodynamics, the linear stiffness and the damping coefficients, and it can be determined via the stability analysis of linear derived equations of the harvester. Therefore, the dimensionless terms are deduced based on above magneto-electro-aeroelastic governing equations, as follows:

$$
\begin{aligned}
& \omega_{h}=\sqrt{\frac{K_{1}}{m_{F}}}, \omega_{\alpha}=\sqrt{\frac{K_{\alpha}}{I_{p}}}, \bar{\omega}=\frac{\omega_{\alpha}}{\omega_{h}}, \bar{h}=\frac{h}{b}, \bar{\alpha}=\alpha, \bar{\eta}=\frac{\theta}{m_{F} \omega_{h}^{2} b} V, \\
& \beta=\frac{M}{m_{F}}, \mu=\frac{m_{F}}{\pi \rho b^{2}}, U_{n o n}=\frac{U}{\omega_{h} b}, \tau=\frac{U t}{b}, R_{3}=\frac{K_{3} b^{2}}{K_{1}}, \\
& R_{\text {mag } 1}=\frac{K_{\text {mag } 1}}{K_{1}}, R_{\text {mag } 3}=\frac{K_{\text {mag } 3} b^{2}}{K_{1}}, \bar{C}_{e q}=\frac{C_{e q} \omega_{h}{ }^{2} m_{F}}{\theta^{2}}, \bar{R}=\frac{\theta^{2}}{\omega_{h} m_{F}} R
\end{aligned}
$$

Based on Wagner function approximation of Theodorsen linear aerodynamic theory, the state-space expression of the linear derived equations is obtained, as follows:

$$
\left\{\begin{array}{l}
\bar{q}^{\prime}(\tau) \\
\bar{q}^{\prime \prime}(\tau) \\
\bar{\eta}^{\prime}(\tau) \\
\bar{w}^{\prime}(\tau)
\end{array}\right\}=\left[\begin{array}{cccc}
0_{2 \times 2} & I_{2 \times 2} & 0_{2 \times 1} & 0_{2 \times 2} \\
-\bar{M}^{-1} \bar{K} & -\bar{M}^{-1} \bar{C} & -\bar{M}^{-1} \bar{\Theta} & -\bar{M}^{-1} \bar{G} \\
0_{1 \times 2} & \left\{\frac{1}{\bar{C}_{p}}, 0\right\} & -\frac{1}{\bar{C}_{p} \bar{R} U_{n o n}} & 0_{1 \times 2} \\
\bar{E}_{q} & \bar{E}_{q d} & 0_{2 \times 1} & \bar{E}_{w}
\end{array}\right]\left\{\begin{array}{l}
\bar{q}(\tau) \\
\bar{q}^{\prime}(\tau) \\
\bar{\eta}(\tau) \\
\bar{w}(\tau)
\end{array}\right\}
$$

where $\bar{q}(\tau)=\{\bar{h}(\tau), \bar{\alpha}(\tau)\}$ is the dimensionless displacement of the airfoil. $\bar{\eta}(\tau)$ is the dimensionless 
output voltage. $\bar{w}(\tau)=\left\{w_{1}(\tau), w_{2}(\tau)\right\}$ is the state variable of the aerodynamic force.

By far, the flutter stability analysis of NMFAEH is equivalent to the eigenvalue analysis of Eq. (14). The root loci can be drawn with respect to the wind speed. The sign of the real part of the eigenvalue roots under the given wind speed can indicate the stability of the harvester. It may increase the wind speed carefully, and the cut-in wind speed can be found once the real part of the eigenvalue roots changes from negative to positive for the first time. One branch of the root loci will cross the imaginary axis from the left half complex plane to the right half. After free decay vibration test [47] and preliminary simulation, the modal damping ratio is set as $\xi_{h}=0.001$ in the theoretical analysis and simulation in this paper. The details of the geometrical and material properties of the presented harvester are shown in Table 1.

Table 1. The geometrical and material properties

\begin{tabular}{|c|c|c|c|}
\hline Properties & Symbol & Value & Units \\
\hline \multicolumn{4}{|c|}{ Middle beam } \\
\hline Length & $L_{b}$ & 17.5 & $\mathrm{~mm}$ \\
\hline Width & $w_{b}$ & 25 & $\mathrm{~mm}$ \\
\hline Thickness & $t_{b}$ & 0.28 & $\mathrm{~mm}$ \\
\hline Density & $\rho_{b}$ & 7850 & $\mathrm{~kg} / \mathrm{m}^{3}$ \\
\hline Young's modulus & $E_{b}$ & 212 & Gpa \\
\hline \multicolumn{4}{|c|}{ Piezoelectric Patches } \\
\hline Length & $L_{p}$ & 42 & $\mathrm{~mm}$ \\
\hline Width & $w_{p}$ & 25 & $\mathrm{~mm}$ \\
\hline Thickness & $t_{p}$ & 0.26 & $\mathrm{~mm}$ \\
\hline Density & $\rho_{p}$ & 7500 & $\mathrm{~kg} / \mathrm{m}^{3}$ \\
\hline Modulus, Open circuit & $c_{11}^{E}$ & 121 & Gpa \\
\hline Piezoelectric constant & $e_{31}$ & 5.4 & $\mathrm{C} / \mathrm{m}^{2}$ \\
\hline Constant strain permittivity & $\varepsilon_{33}^{s}$ & 7.3455 & $\mathrm{nF} / \mathrm{m}$ \\
\hline Copper substrate length & $L_{c}$ & 42 & $\mathrm{~mm}$ \\
\hline Copper substrate width & $w_{c}$ & 30 & $\mathrm{~mm}$ \\
\hline Copper substrate thickness & $t_{c}$ & 0.2 & $\mathrm{~mm}$ \\
\hline Copper density & $\rho_{c}$ & 8920 & $\mathrm{~kg} / \mathrm{m}^{3}$ \\
\hline Copper Young's modulus & $E_{c}$ & 106 & Gpa \\
\hline \multicolumn{4}{|c|}{ NACA 0012 Profile Airfoil } \\
\hline Airfoil mass & $m_{F}$ & 16 & $\mathrm{~g}$ \\
\hline Semichord & $b$ & 40 & $\mathrm{~mm}$ \\
\hline Mass moment of inertia & $I_{p}$ & 9.684 & $\mathrm{~kg} / \mathrm{mm}^{2}$ \\
\hline Static unbalance & $x_{\alpha}$ & 0.358 & \\
\hline
\end{tabular}




\begin{tabular}{cccc}
\hline Mass of tip magnets and connections & $m_{T}$ & 10.6 & $\mathrm{~g}$ \\
\hline \multicolumn{4}{c}{ Circuit } \\
\hline Load resistance & $R$ & 10 & $\mathrm{M} \Omega$ \\
\hline
\end{tabular}

Based on the root-locus method shown in Figure 6, the real part of the eigenvalues represents to the aeroelastic coupled damping coefficient of each degree of freedom, and the imaginary part represents to the aeroelastic coupled frequencies. The flutter boundary can be determined by the changing regularity of the real part's plus-minus sign, while the flutter frequency is obtained from the imaginary part of the corresponding eigenvalue. It is found from Figure 6(a) that the real part's sign of the eigenvalue in the plunging freedom becomes positive after the cut-in wind speed of $U_{f}=0.997 \mathrm{~m} / \mathrm{s}$, while the real parts' sign of the eigenvalue in the pitching, voltage and aerodynamic force are still negative. This critical wind speed is defined to be the flutter speed of the NMFAEH. As shown in Figure 6 (b), the imaginary part of the eigenvalue in the plunging decreases along with the increase of the wind speed. The pitching frequency increases because of the additional stiffness caused by aerodynamic loads along with the increase of the wind speed. Meanwhile, the imaginary part of the eigenvalues in the plunging and pitching state-space equations are close to each other. In addition, the imaginary part of the eigenvalues in the voltage state-space equation is always be zero. According to the flutter frequency coincidence theory $[44,45]$, the flutter of the NMFAEH can be classified as the airfoil plunging and pitching coupled mode. The equilibrium position becomes unstable beyond the cut-in wind speed. In this case, the NMFAEH enters into limit cycle oscillations and efficiently harvest energy from the airflow.
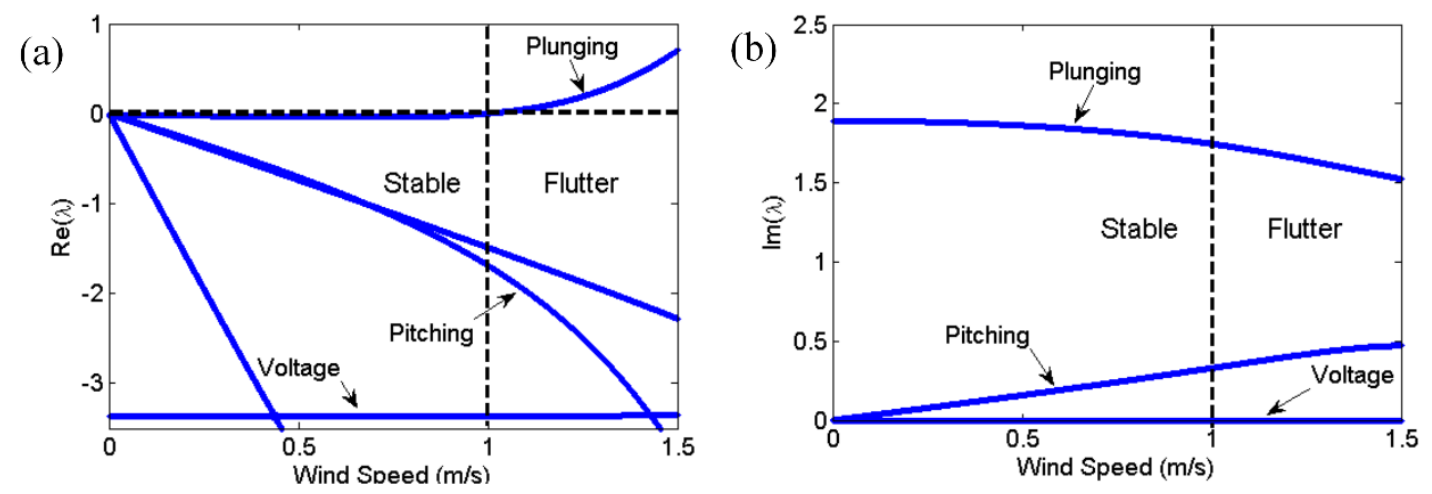

Figure 6. Eigenvalue analysis versus the wind speed for NMFAEH: (a) The real part; (b) the imaginary part.

In order to deeply analyze the influence of the nonlinear magnetic force, the theoretical analysis of the FAEH is provided in Figure 7, in which the nonlinear magnetic force is cancelled while remaining other 
configurations. Its cut-in wind speed can be obtained to be $U_{f}=1.913 \mathrm{~m} / \mathrm{s}$, which is much higher than that of the NMFAEH. It is found from the equivalent stiffness above that softened magnetic force decreases the plunging frequency, therefore, the plunging and pitching mode is more likely to be coupled as shown in Figure 6 (b) and Figure 7 (b). As a result, the flutter of the NMFAEH occurs at the lower wind speed, which is almost $50 \%$ lower than that of the FAEH. This indicates that the NMFAEH can efficiently harvest energy from a lower wind speed range.
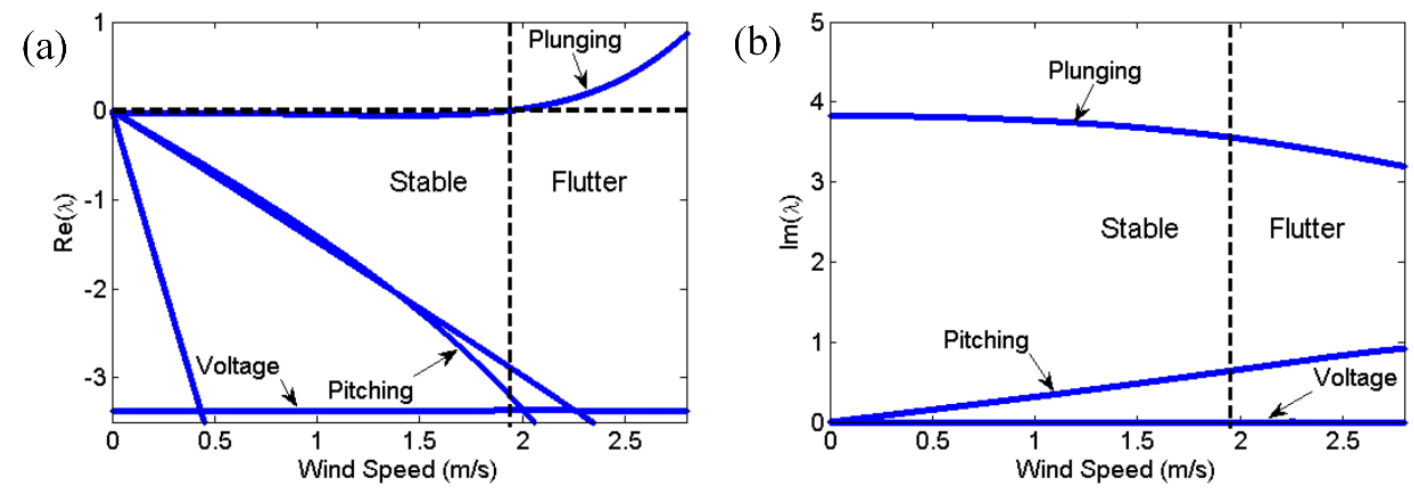

Figure 7. Eigenvalue analysis versus the wind speed for FAEH: (a) The real part; (b) the imaginary part.

\subsection{Time-domain simulation}

By substituting Eqs. (9) - (12) into Eqs. (5) - (7), the nonlinear magneto-electro-aeroelastic governing equations for the NMFAEH are deduced. Both the NMFAEH and the FAEH are numerically simulated using the Runge-Kutta method based on MATLAB software to predict the dynamic response and the output voltage of the harvesters.

Figure 8 shows the effect of the load resistance for different wind speeds cases on energy harvesting performance. It can be obtained from Figure 8 (a) that for each wind speed case the output voltage amplitude firstly increases with the increasing of the load resistance then tends to a constant value. In figure 8 (b), the optimal load resistance which leads to the maximum power output can be found between $10^{6} \Omega$ and $10^{7} \Omega$ (the value is $R_{o p t}=\frac{1}{C_{p} \omega}$ as given in Ref. [29], where $\omega$ is the angular frequency of flutter limit cycle oscillations (LCOs)). For flutter-based magnetic-coupled energy harvesting, the aerodynamic force and the magnetic force will bring additional equivalent stiffness and damping to the harvester. Therefore, the optimal load resistance 
shifts to a smaller one with the increasing of the wind speed as a result of the increasing of the LCO frequency shown in Figure 8 (c). Meanwhile, in Figure 8 (d), the optimal load resistance slightly leads to a 2\% increase of linear flutter speed (with respect to that of $10^{9} \Omega$ nearly open-circuit condition) due to the shunt damping effect of the piezoelectric transducer [5]. However, the value of the electromechanical coupling coefficient in this study is $1.36 \times 10^{-2} \mathrm{mN} / \mathrm{V}$ which is a weakly coupled factor. Therefore, the load resistance has no considerable impact on linear flutter speed and dynamic responses in this study. This was also verified by Refs. [27, 29, 31].
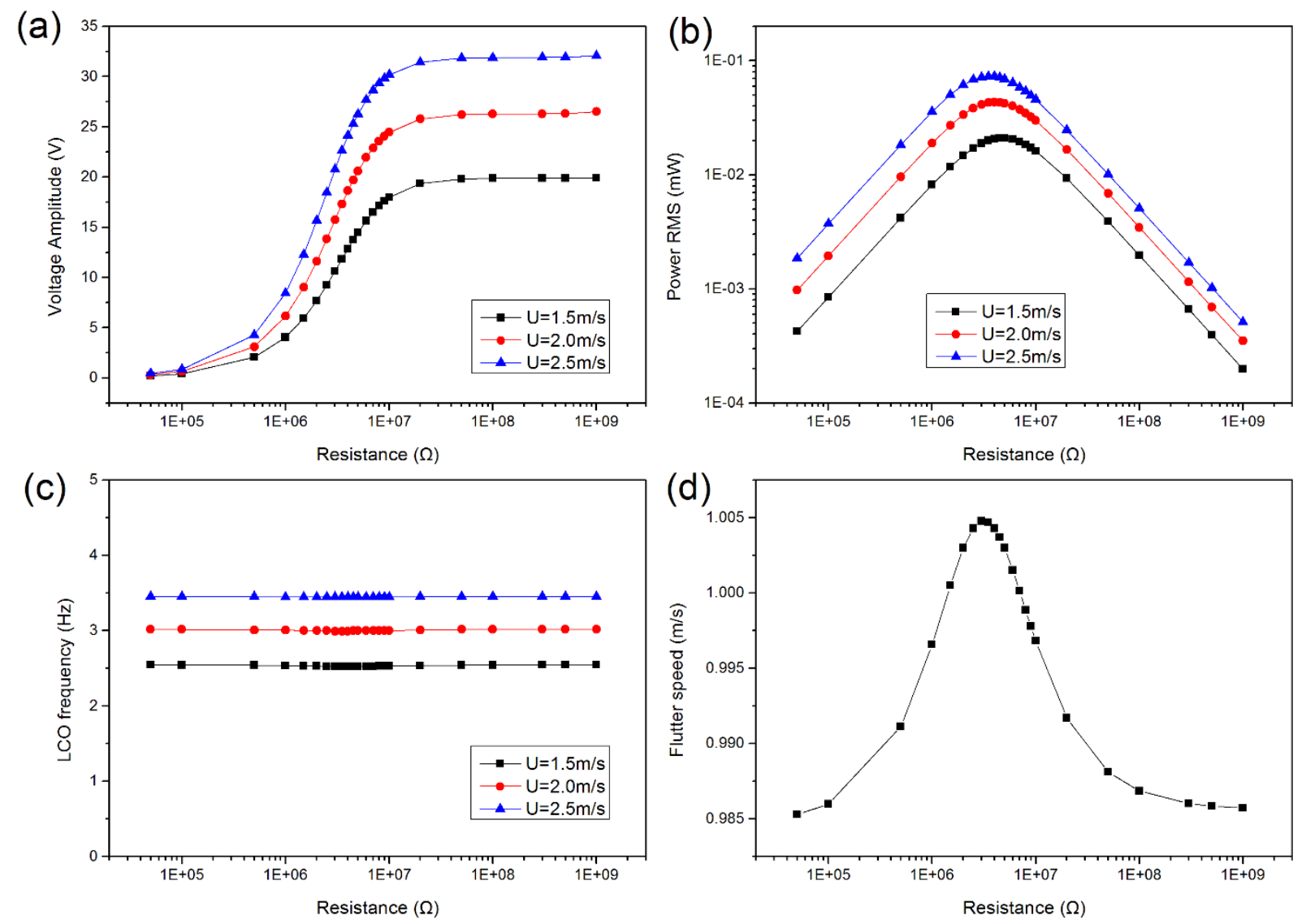

Figure 8. Variations of three wind speed cases: (a) Output voltage amplitude; (b) power RMS;

(c) flutter LCO frequency; (d) flutter speed versus load resistance.

Figure 9 shows the time-domain output voltage of the NMFAEH and the FAEH for different wind speeds, and each case begins from the same initial condition. In Figure 9 (a), the wind speed is lower than the cut-in wind speed of both the NMFAEH and the FAEH. It is found that the output voltage finally attenuates to nearly zero. When the wind speed is higher than the cut-in wind speed of the NMFAEH while being lower than that of the FAEH, it is observed in Figure 9 (b) that the movement of NMFAEH transforms into stable limit cycle 
oscillations which will lead to the large-amplitude output voltage. However, the responses are dissipated and the output voltage of the FAEH are relatively small at the same wind speed.

The output voltage increases along with the increase of the wind speed. As shown in figure 9 (b), (c) and (d), the output voltage amplitude of the NMFAEH is $12.48 \mathrm{~V}, 25.83 \mathrm{~V}$ and $32.54 \mathrm{~V}$ for $U=1.1 \mathrm{~m} / \mathrm{s}, U=2.0 \mathrm{~m} / \mathrm{s}$ and $U=2.6 \mathrm{~m} / \mathrm{s}$, respectively. When the wind speed exceeds the cut-in wind speed of the FAEH, it begins to efficiently work. The output voltage amplitude of the FAEH is respectively $17.45 \mathrm{~V}$ and $30.34 \mathrm{~V}$ for $U=2.0 \mathrm{~m} / \mathrm{s}$ and $U=2.6 \mathrm{~m} / \mathrm{s}$, as shown in Figure 9 (c) and (d), which is $8.38 \mathrm{~V}$ and $2.2 \mathrm{~V}$ smaller than that of the NMFAEH. The comparison of output voltage between the NMFAEH and the FAEH can be more concisely observed by the trajectory obit shown in Figure 10. Then the output power of harvester can be calculated by Eq. (8). The bifurcation diagram is illustrated in Figure 11. It is evident that the NMFAEH can produce larger output voltage and power in the low wind speed range of $1 \mathrm{~m} / \mathrm{s} \sim 2.8 \mathrm{~m} / \mathrm{s}$.

Briefly speaking, from the time-domain simulations, it is found that the cut-in wind speed of the NMFAEH is lower than that of the FAEH. Meanwhile, the output voltage of the NMFAEH is larger than the latter at the low wind speed range. This further verifies that the nonlinear magnetic force can be introduced to adjust the characteristics of the flutter-based harvesters and enhance its energy harvesting efficiency in the low wind speed range. 
(a)
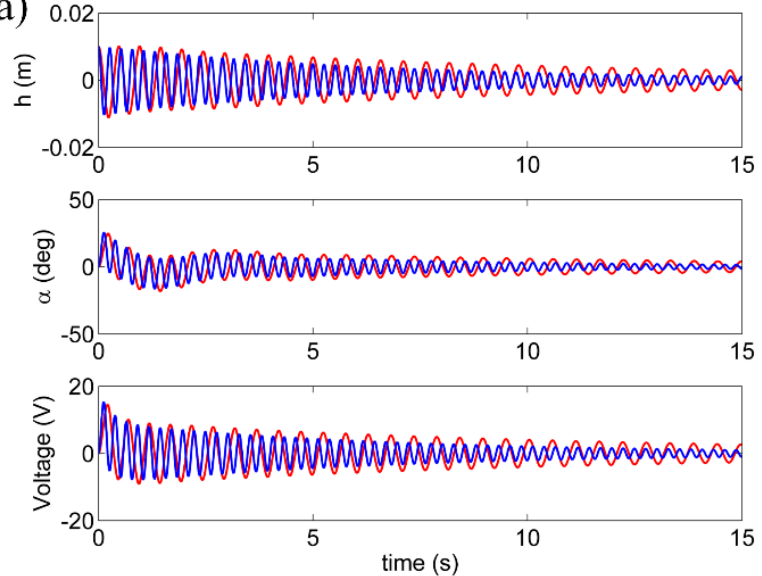

(c)
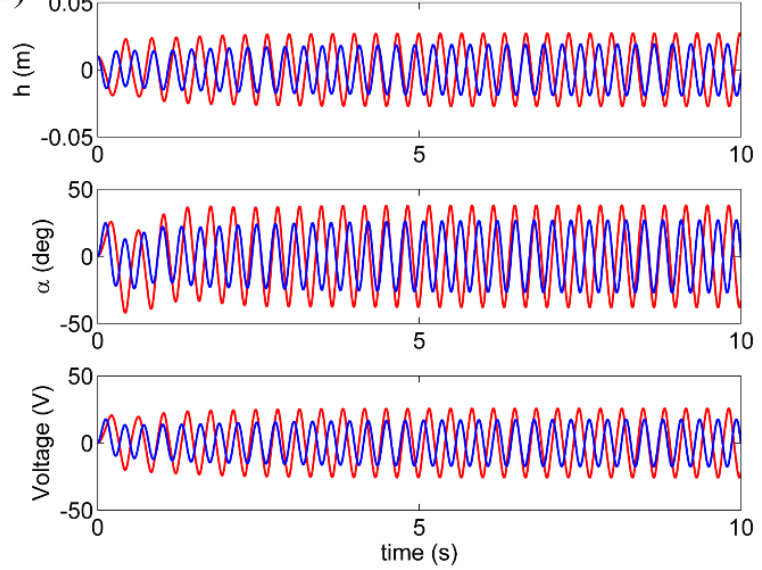

(b)
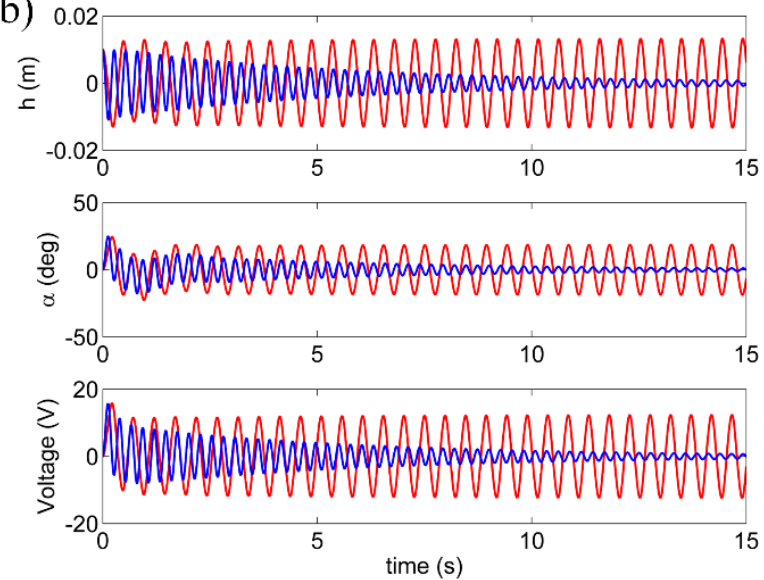

(d)
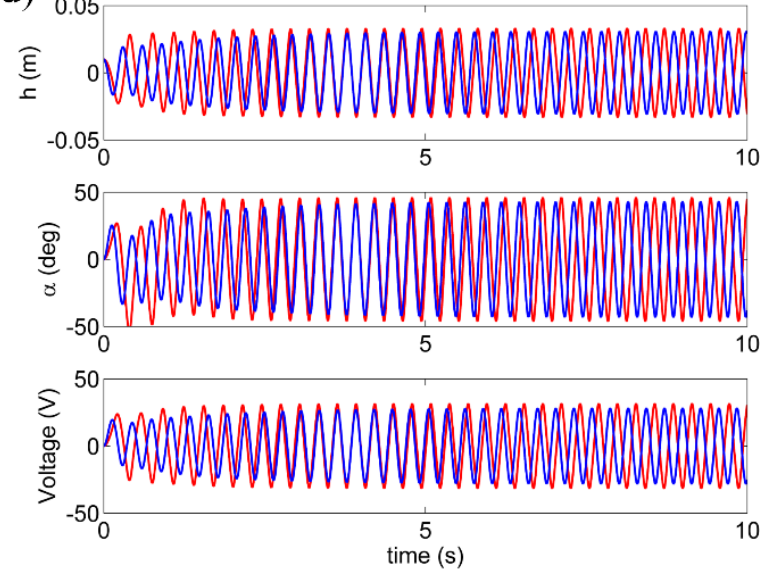

Figure 9. Numerical time-domain output voltage of the NMFAEH (red) and the FAEH (blue) at the wind speed: (a) $U=0.7 \mathrm{~m} / \mathrm{s}$; (b) $U=1.1 \mathrm{~m} / \mathrm{s}$; (c) $U=2.0 \mathrm{~m} / \mathrm{s}$; (d) $U=2.6 \mathrm{~m} / \mathrm{s}$.
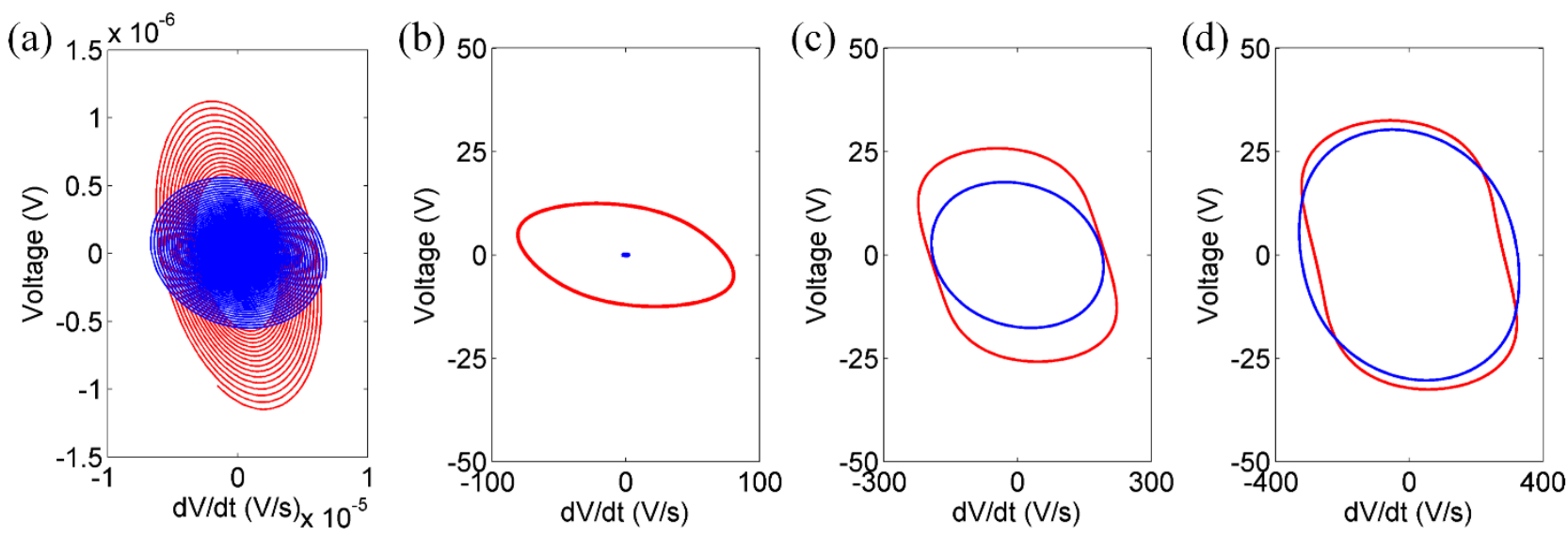

Figure 10. Trajectory plot of the output voltage of the NMFAEH (red) and the FAEH (blue) at the wind speed: (a) $U=0.7 \mathrm{~m} / \mathrm{s}$; (b) $U=1.1 \mathrm{~m} / \mathrm{s}$; (c) $U=2.0 \mathrm{~m} / \mathrm{s}$; (d) $U=2.6 \mathrm{~m} / \mathrm{s}$. 

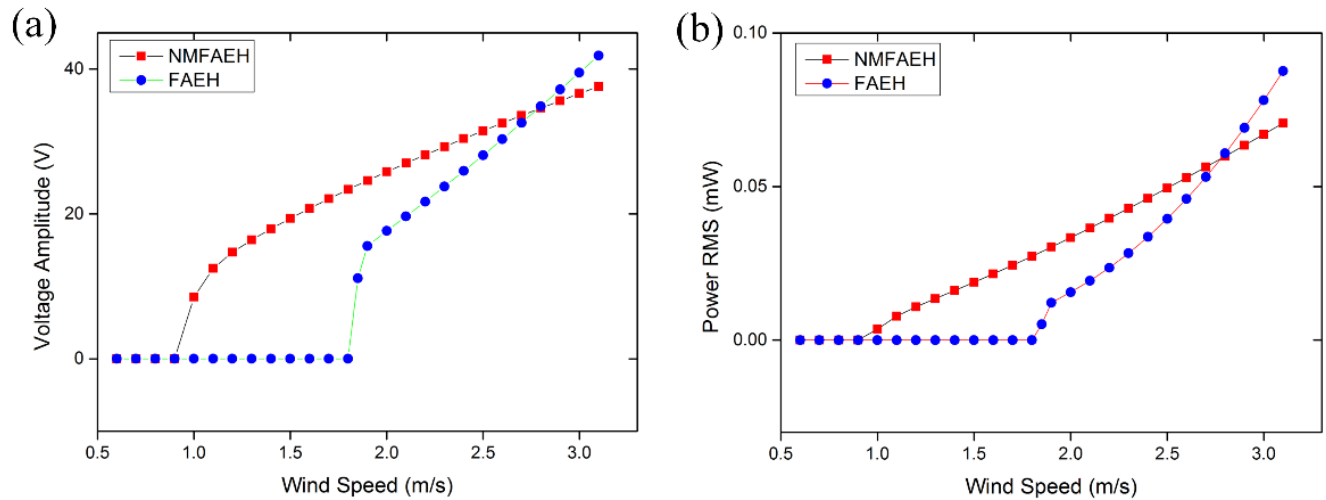

Figure 11. Simulation results between NMFAEH and FAEH: (a) Output voltage and (b) power RMS.

\section{Experimental verification}

\subsection{Wind tunnel experimental verification}

In order to verify the theoretical model and analytical analysis, experimental validation is carried out in this section. Front view and side view of experimental setup are shown in Figure 12 (a) and (b), respectively. The middle substrate of the piezoelectric beam is made of stainless steel. Two piezoelectric patches with copper substrates are pasted to the clamped end of the cantilever beam by using the epoxy resin. The two-dimensional airfoil is made of balsa-wood and its profile is NACA0012. The part of the hinged connection is fabricated by a 3-D printer. The piezoelectric beam is clamped at a base aluminum alloy structure. The NMFAEH is fixed at the test chamber of a wind tunnel. The length direction of the piezoelectric beam is in the same direction of the wind flow. The gravitational force of the two-dimensional airfoil has no influence on the NMFAEH, because the piezoelectric beam horizontally oscillates. A precision anemometer (Testo 416) is installed in the entry of the wind flow to timely measure the wind speed. An oscilloscope (Tektronix ${ }^{\text {TM }}$ TDS 1002) is used to display and measure the output voltage. 

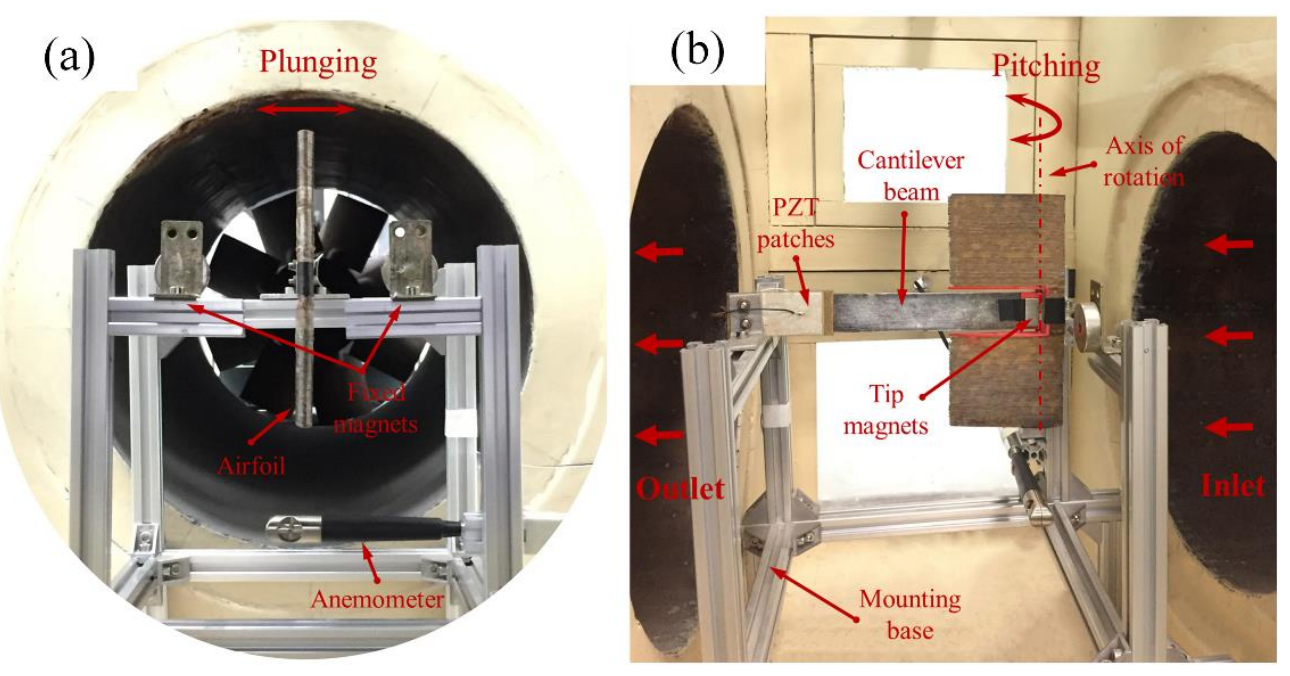

Figure 12. Experimental setup: (a) Front view; (b) side view.

Based on the stability analysis and the numerical simulation, the wind speed range of $0.6 \mathrm{~m} / \mathrm{s}-3.1 \mathrm{~m} / \mathrm{s}$ is selected in experiments. Figure 13 shows the numerical and experimental output voltage and the mean square root of the output power along with the increase of the wind speed of the NMFAEH. It is found in Figure 13 that the equilibrium position is stable when the wind speed is smaller than the cut-in wind speed and the oscillation of the NMFAEH attenuates to zero. The real part of the eigenvalues of the NMFAEH increases to a positive value when the wind speed is larger than the cut-in wind speed. In this case, the equilibrium position becomes unstable from stable status. Self-sustained vibration occurs in the NMFAEH and the vibration promptly surges. With the increase of the vibration amplitude, the attractive nonlinear magnetic force converts into repulsion and hardens the stiffness of the harvester. Therefore, the stable limit cycle oscillation grows out. The voltage bifurcation diagram and the power bifurcation diagram of the NMFAEH show supercritical flutter characteristics, corresponding to the supercritical Hopf bifurcation of the nonlinear system. The NMFAEH begins to efficiently harvest energy from wind flows at the wind speed of $1.1 \mathrm{~m} / \mathrm{s}$ and produce large-amplitude output voltage. This can be used to explain the working mechanism of the NMFAEH.

Figure 14 shows comparison of the flutter frequency in both simulations and experiments. It is found that the flutter frequency of the NMFAEH increases slightly along with the increase of the wind speed. A very good agreement between numerical simulations and experimental results is shown in Figures 13 and 14. The error may be caused by the measurement and identification of the nonlinear magnetic force. 
(a)

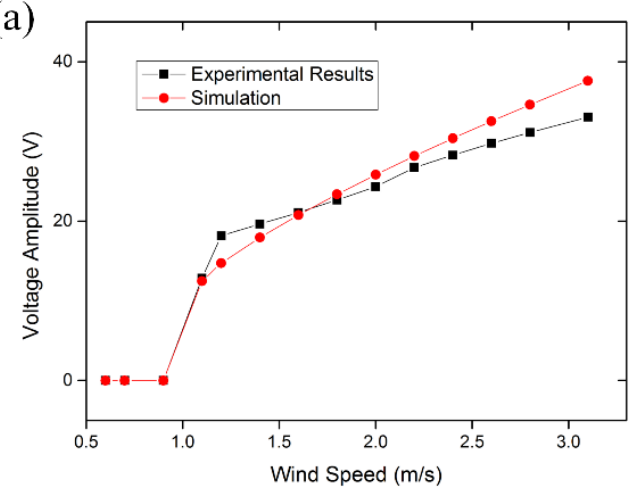

(b)

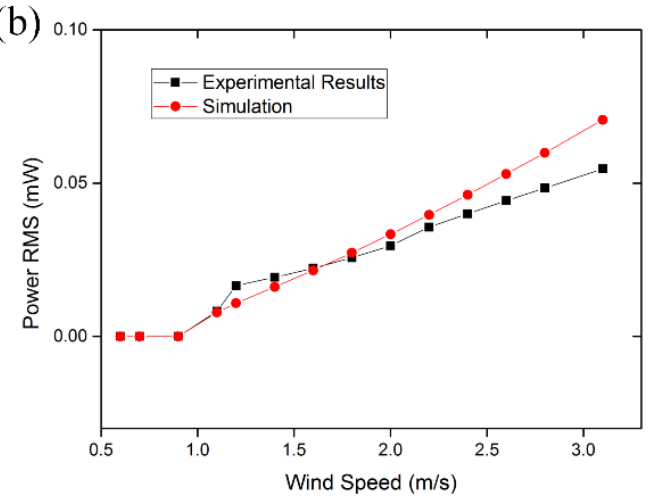

Figure 13. Bifurcation diagrams versus wind speeds: (a) Output voltage and (b) power RMS.

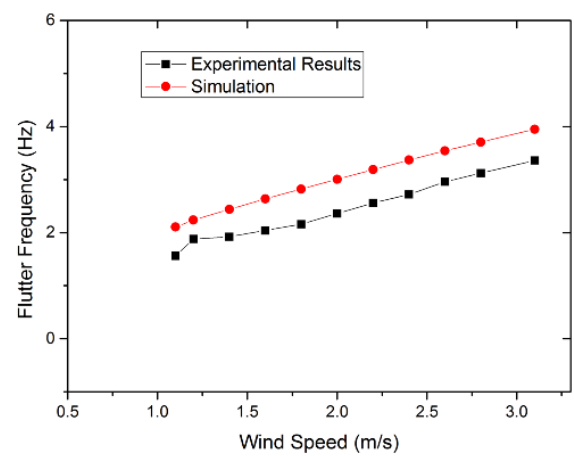

Figure 14. Comparison of the flutter frequency in simulations and experiments.

\subsection{Comparison with the FAEH}

Figure 15 and 16 show the experimental comparison of the time-domain output voltage and the corresponding equivalent trajectory obit of the NMFAEH and the FAEH. As shown in Figure 15 (a), it is found that the NMFAEH already enters into limit cycle oscillations at the wind speed of $U=1.2 \mathrm{~m} / \mathrm{s}$, and the output voltage amplitude is $14.72 \mathrm{~V}$. However, the oscillation orbit of the FAEH almost decays to zero, and it cannot efficiently harvest energy. As the wind speed increases to $1.9 \mathrm{~m} / \mathrm{s}$ in theoretical analysis, the FAEH begins to its limit cycle oscillations and output large-amplitude voltage in experiments. Meanwhile, its output voltage is still smaller than that of the NMFAEH. For example, the output voltage amplitude of the NMFAEH is $28.28 \mathrm{~V}$ and $31.13 \mathrm{~V}$ at the wind speed of $2.4 \mathrm{~m} / \mathrm{s}$ and $2.8 \mathrm{~m} / \mathrm{s}$, respectively, as shown in Figure 15 (b) and Figure 16 (a). Meanwhile, the output voltage amplitude of the FAEH is $18.86 \mathrm{~V}$ and $27.40 \mathrm{~V}$ respectively at the above two wind speeds. Compared with the FAEH, the output voltage increases to $149.9 \%$ and $113.6 \%$, respectively. The 
difference in the output voltage between the NMFAEH and the FAEH is gradually decreasing along with the increase of the wind speed, which is affected by the nonlinear magnetic force. In the relative small wind speed range, the tip displacement of the NMFAEH mainly locates in the attractive magnetic force range. As the wind speed increases, the aerodynamic force will excite it into the repulsive range in which the nonlinear magnetic force hardens the stiffness.

In order to protect the experimental device, the maximum wind speed is set as $3.1 \mathrm{~m} / \mathrm{s}$. As shown in Figure 16 (b), although the FAEH has a larger output voltage (37.79V) than that of the NMFAEH (33.06V) when the wind speed is $3.1 \mathrm{~m} / \mathrm{s}$, the observed deformation of the cantilever beam of the former is very large which may damage the piezoelectric ceramics of the harvester. This indicates that one can reduce the structural stiffness of the FAEH to decrease the cut-in wind speed and enhance the energy harvesting efficiency. However, the excessive structural deformation is dangerous to the harvester when the wind speed is too large. The NMFAEH's smaller deformation at the large wind speed will protect its structure safety.
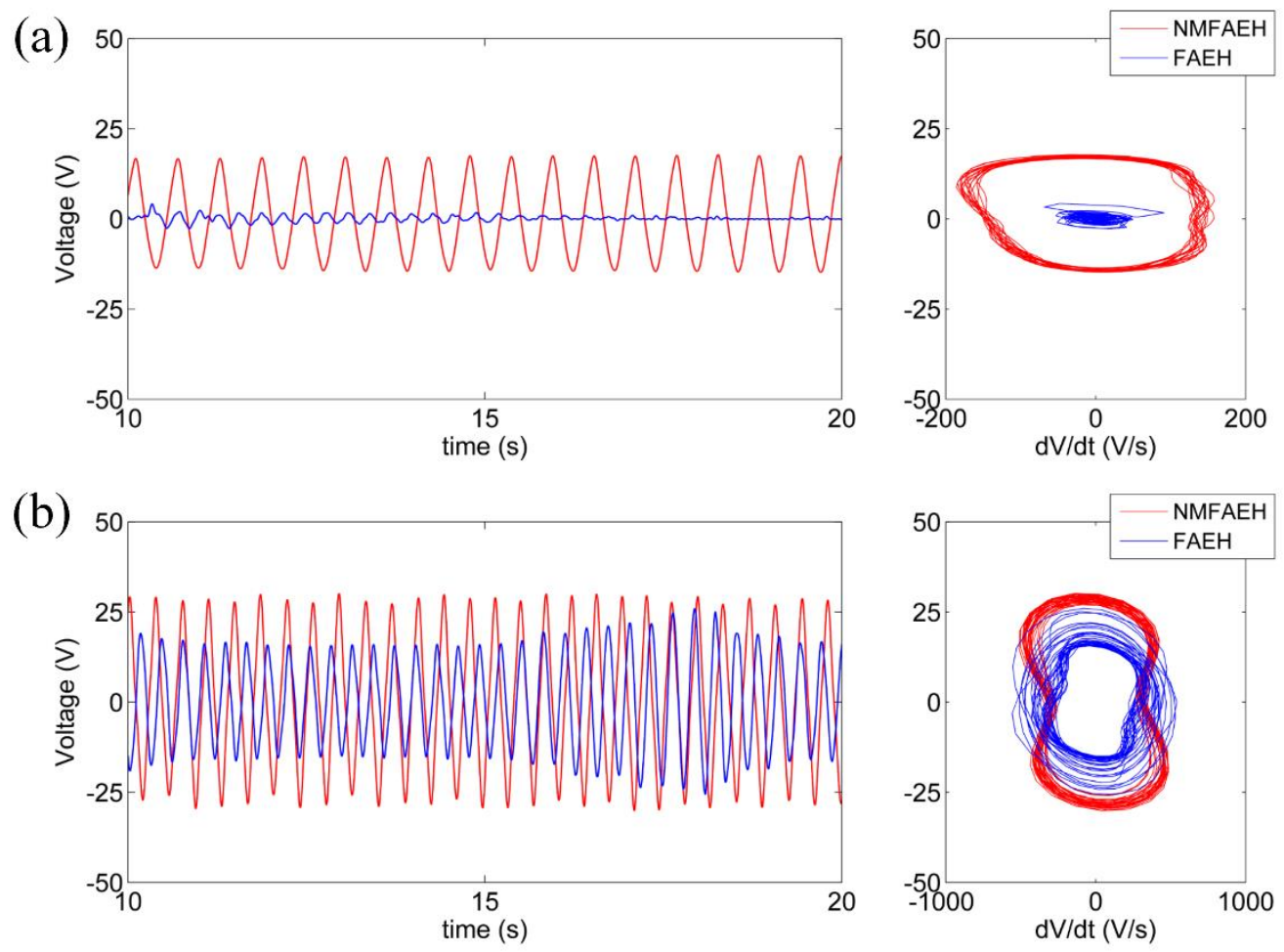

Figure 15. Experimental comparison of the NMFAEH and the FAEH, time history and the orbit of the output voltage: (a) $U=1.2 \mathrm{~m} / \mathrm{s}$; (b) $U=2.4 \mathrm{~m} / \mathrm{s}$. 

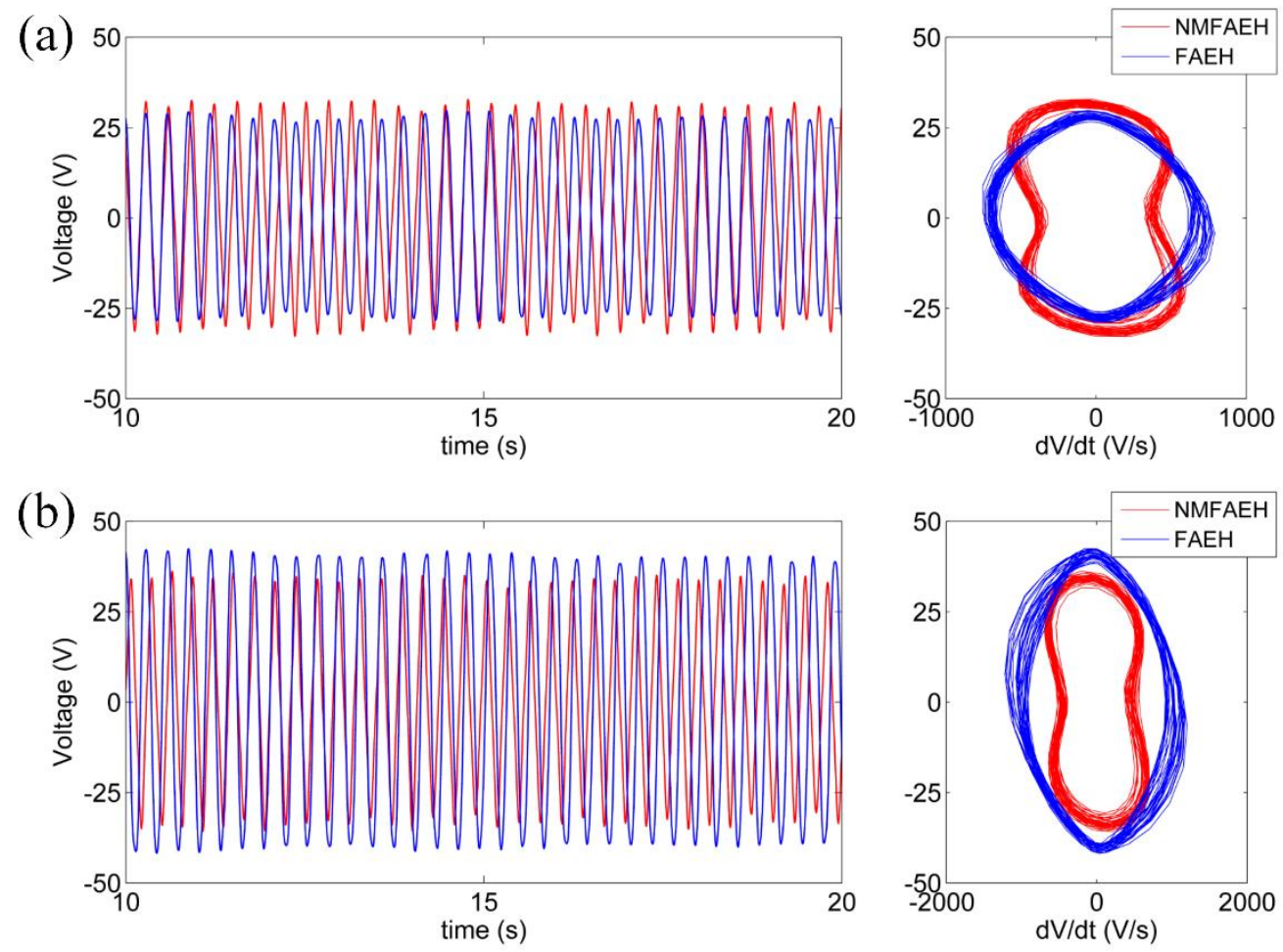

Figure 16. Experimental comparison of the NMFAEH and the FAEH, time history and the orbit of the output voltage: (a) $U=2.8 \mathrm{~m} / \mathrm{s}$; (b) $U=3.1 \mathrm{~m} / \mathrm{s}$.

Figure 17 shows the output voltage amplitude of both the NMFAEH and the FAEH versus wind speed. It is experimentally found that the cut-in wind speed of the NMFAEH is in the vicinity of $1 \mathrm{~m} / \mathrm{s}$, while the cut-in wind speed of the FAEH is about $1.9 \mathrm{~m} / \mathrm{s}$, which are quite consistent with the theoretical results. This demonstrates that the cut-in wind speed can be reduced by the additional nonlinear magnetic force to the harvester. Therefore, the NMFAEH can efficiently harvest energy from the low wind speed range of $1 \mathrm{~m} / \mathrm{s} \sim 1.9$ $\mathrm{m} / \mathrm{s}$, while the efficiency of the FAEH is very low and its output voltage is nearly zero in the same range. More importantly, experimental results show that the NMFAEH has a larger output voltage and a better energy harvesting efficiency than the traditional FAEH in the wind speed range of $1 \mathrm{~m} / \mathrm{s} \sim 2.9 \mathrm{~m} / \mathrm{s}$. The better performance of the NMFAEH can be calculated through a quantitative indicator defined as:

$$
\Sigma=\int_{U_{0}}^{U_{T}} P d U
$$

This indicator denotes to the surrounded area by the power RMS and the wind speed, which can be used to reflect the capability of energy harvesting in the wide wind speed range of $0.6 \mathrm{~m} / \mathrm{s} \sim 3.1 \mathrm{~m} / \mathrm{s}$. The surrounded 
area of the NMFAEH and the FAEH is $0.070 \mathrm{~mW} \cdot \mathrm{m} / \mathrm{s}$ and $0.040 \mathrm{~mW} \cdot \mathrm{m} / \mathrm{s}$, respectively. This shows that the NMFAEH can generate more power for the same wind speed situation. The indicator reveals that the energy harvesting performance with the magnetic force coupling increased to $175 \%$ than that of the FAEH. Therefore, the presented NMFAEH can enhance energy harvesting at low wind speeds. Simulation predictions which are $0.0793 \mathrm{~mW} \cdot \mathrm{m} / \mathrm{s}$ for the NMFAEH and $0.0567 \mathrm{~mW} \cdot \mathrm{m} / \mathrm{s}$ for the FAEH also demonstrate good agreement with experimental results.
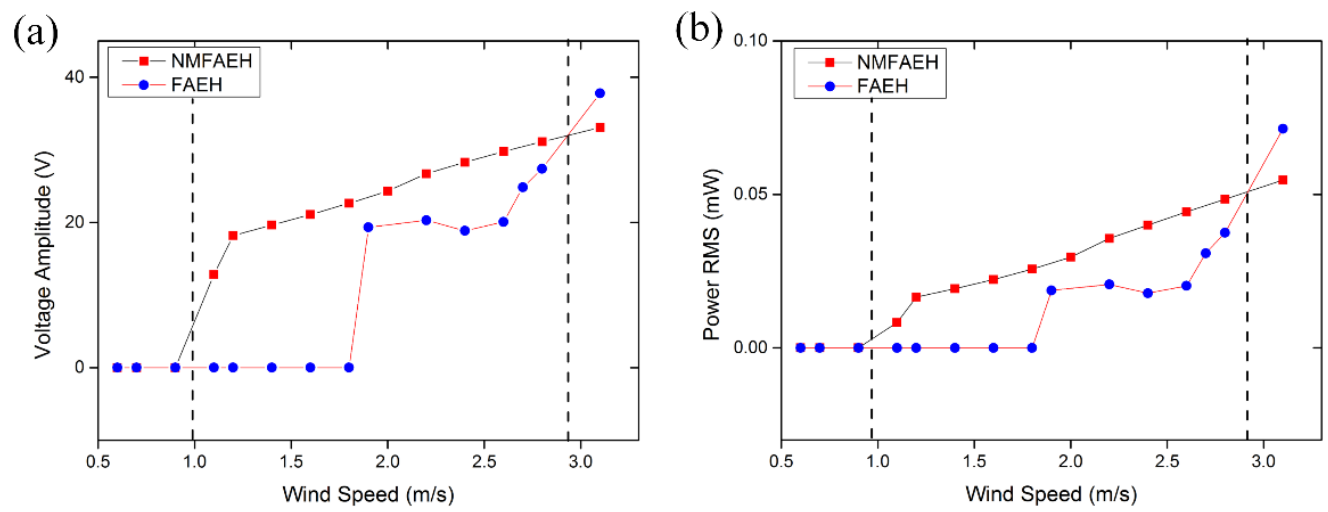

Figure 17. Comparison of bifurcation diagrams between the NMFAEH and the FAEH:

(a) output voltage; (b) power RMS

\section{Conclusions}

In this paper, a novel nonlinear magnetic-coupled flutter-based aeroelastic energy harvester (NMFAEH) is presented. The nonlinear magnetic force is experimentally modeled and the magneto-electro-aeroelastic governing equations of the NMFAEH are theoretically established. The influence of the nonlinear magnetic force on the harvester is analytically investigated. Stability analysis and numerical study show that the NMFAEH has a lower cut-in wind speed for large-amplitude limit cycle oscillations and is more efficient in the low wind speed range than the FAEH. Experimental verifications are performed in the range of $0.6 \mathrm{~m} / \mathrm{s} \sim 3.1$ $\mathrm{m} / \mathrm{s}$ for the NMFAEH and the FAEH. The results demonstrate that the NMFAEH has a low cut-in wind speed (about $1 \mathrm{~m} / \mathrm{s}$ ) which is almost $50 \%$ lower than that of the FAEH. This is very beneficial for aeroelastic energy harvesting at low wind speed conditions. Moreover, in the wind speed range of $1 \mathrm{~m} / \mathrm{s} \sim 2.9 \mathrm{~m} / \mathrm{s}$, the output voltage and power of the NMFAEH is larger. The comprehensive indicator shows that the presented NMFAEH enhanced energy harvesting performance to $175 \%$, comparing to the FAEH, which indicates that the nonlinear 
magnetic force broadens the effective wind speed range and improves the energy harvesting efficiency of flutterbased aeroelastic energy harvester. Meanwhile, the results demonstrate that the NMFAEH is more capable to harvest energy in the low wind speed range.

\section{Acknowledgements}

This project has been supported by the National Natural Science Foundation of China (Grant Nos. 11672240, 11072198, 11502208).

\section{Appendix A}

In the presented model, the piezoelectric beam can be taken as a stepped beam with two different sections as shown in Figure 2. Therefore, we can assume the beam mode shapes of the two different parts as $\phi_{a}(x)$ and $\phi_{b}(x)$ separately, as follows:

$$
\begin{array}{ll}
\phi_{a}(x)=C_{a 1} \cos \left(\beta_{a} x\right)+C_{a 2} \sin \left(\beta_{a} x\right)+C_{a 3} \cosh \left(\beta_{a} x\right)+C_{a 4} \sinh \left(\beta_{a} x\right) & , x \in\left[L_{p}, L_{b}\right] \\
\phi_{b}(x)=C_{b 1} \cos \left(\beta_{b} x\right)+C_{b 2} \sin \left(\beta_{b} x\right)+C_{b 3} \cosh \left(\beta_{b} x\right)+C_{b 4} \sinh \left(\beta_{b} x\right) & , x \in\left[0, L_{p}\right]
\end{array}
$$

At the joint part, the continuous condition of the displacement, the angle of rotation, the bending moment and the shear force, combined with the boundary conditions simultaneously are defined as:

$$
\begin{gathered}
D_{a} \phi_{a}^{\prime \prime}\left(L_{b}\right)=0, D_{a} \phi_{a}^{\prime \prime \prime}\left(L_{b}\right)=0, \\
\phi_{b}(0)=0, \phi_{b}^{\prime}(0)=0, \\
\phi_{a}\left(L_{p}\right)=\phi_{b}\left(L_{p}\right), \phi_{a}^{\prime}\left(L_{p}\right)=\phi_{b}^{\prime}\left(L_{p}\right), \\
D_{a} \phi_{a}^{\prime \prime}\left(L_{p}\right)=D_{b} \phi_{b}^{\prime \prime}\left(L_{p}\right), D_{a} \phi_{a}^{\prime \prime \prime}\left(L_{p}\right)=D_{b} \phi_{b}^{\prime \prime \prime}\left(L_{p}\right) .
\end{gathered}
$$

where $\quad \beta_{a}=\sqrt{\frac{\omega}{D_{a} / m_{a}}}$ and $\beta_{b}=\sqrt{\frac{\omega}{D_{b} / m_{b}}} \cdot \omega$ is the natural frequency of the beam. $m_{a}$ and $m_{b}$ are the mass per unit length of the first and second parts of the piezoelectric beam, respectively. $D_{a}$ and $D_{b}$ are the bending stiffness of the first and second parts of the piezoelectric beam, respectively. By substituting the assumed mode shapes into Eqs. (A3)-(A6), the orthogonal modes of the variable cross-section beam in this paper can be obtained. 


\section{References}

[1] Erturk A and Inman D J 2011 Piezoelectric Energy Harvesting (Chichester: Wiely)

[2] Zhou S, Cao J, Litak G and Lin J 2018 Numerical analysis and experimental verification of broadband tristable energy harvesters TM-Tech. Mess. (https://doi.org/10.1515/teme-2017-0076)

[3] Wang F, Sun X and Xu J 2018 A novel energy harvesting device for ultralow frequency excitation Energy $151250-260$.

[4] Yao M H and Zhang W 2014 Multi-Pulse chaotic motions of high-dimension nonlinear system for a laminated composite piezoelectric rectangular plate Meccanica 49 365-392.

[5] Abdelkefi A 2016 Aeroelastic energy harvesting: A review Int. J. Eng. Sci. 100 112-135.

[6] Hobeck J D and Inman D J 2016 Dual cantilever flutter: experimentally validated lumped parameter modeling and numerical characterization J. Fluid Struct. 61 324-338.

[7] Xiang H J, Zhang Z W, Shi Z F and Li H 2018 Reduced-order modeling of piezoelectric energy harvesters with nonlinear circuits under complex conditions Smart Mater. Struct. 27045004.

[8] Chen L Q and Jiang W A 2015 Internal resonance energy harvesting J. Appl. Mech. 82031004.

[9] Ghavami M, Azizi S and Ghazavi M R 2018 On the dynamics of a capacitive electret-based microcantilever for energy harvesting Energy 153 967-976.

[10] Zhou S and Zuo L 2018 Nonlinear dynamic analysis of asymmetric tristable energy harvesters for enhanced energy harvesting Commun. Nonlinear Sci. 61 271-284.

[11] Yan B, Zhang S, Zhang X, Wang K and Wu C 2017 Self-powered electromagnetic energy harvesting for the low power consumption electronics: Design and experiment Int. J. Appl. Electro. 54 165-175.

[12] Wang W, Cao J, Mallick D, Roy S and Lin J 2018 Comparison of harmonic balance and multi-scale method in characterizing the response of monostable energy harvesters Mech. Syst. Signal Pr. 108 252-261.

[13] Akaydin H D, Elvin N and Andreopoulos Y 2012 The performance of a self-excited fluidic energy harvester Smart Mater. Struct. 21025007.

[14] Goushcha O, Elvin N and Andreopoulos Y 2014 Interactions of vortices with a flexible beam with applications in fluidic energy harvesting Appl. Phys. Lett. 104021919.

[15] Dai H L, Abdelkefi A, Yang Y and Wang L 2016 Orientation of bluff body for designing efficient energy 
harvesters from vortex-induced vibrations Appl. Phys. Lett. 108053902.

[16] Zhang B, Song B, Mao Z, Tian W and Li B 2017 Numerical investigation on VIV energy harvesting of bluff bodies with different cross sections in tandem arrangement Energy 133 723-736.

[17] Weinstein L A, Cacan M R, So P M and Wright P K 2012 Vortex shedding induced energy harvesting from piezoelectric materials in heating, ventilation and air conditioning flows Smart Mater. Struct. 21045003.

[18] Zhang M, Zhao G and Wang J 2017 Study on Fluid-Induced Vibration Power Harvesting of Square Columns under Different Attack Angles Geofluids 2 1-18.

[19] Zhou S and Wang J 2018 Dual serial vortex-induced energy harvesting system for enhanced energy harvesting AIP Adv. 8075221.

[20] Dai H L, Yang Y W, Abdelkefi A and Wang L 2018 Nonlinear analysis and characteristics of inductive galloping energy harvesters Commun. Nonlinear Sci. 59 580-591.

[21] Vicente-Ludlam D, Barrero-Gil A and Velazquez A 2015 Enhanced mechanical energy extraction from transverse galloping using a dual mass system J. Sound Vib. 339 290-303.

[22] Ewere F, Wang G and Cain B 2014 Experimental investigation of galloping piezoelectric energy harvesters with square bluff bodies Smart Mater. Struct. 23104012.

[23] Zhao L, Tang L and Yang Y 2013 Comparison of modeling methods and parametric study for a piezoelectric wind energy harvester Smart Mater. Struct. 22125003.

[24] Zhao L and Yang Y 2015 Enhanced aeroelastic energy harvesting with a beam stiffener Smart Mater. Struct. 24032001.

[25] Abdelkefi A, Yan Z and Hajj M R 2013 Modeling and nonlinear analysis of piezoelectric energy harvesting from transverse galloping Smart Mater. Struct. 22025016.

[26] Tan T and Yan Z 2016 Analytical solution and optimal design for galloping-based piezoelectric energy harvesters Appl. Phys. Lett. 109253902.

[27] Erturk A, Vieira W G R, De Marqui Jr C and Inman D J 2010 On the energy harvesting potential of piezoaeroelastic systems Appl. Phys. Lett. 96184103.

[28] Li S, Yuan J and Lipson H 2011 Ambient wind energy harvesting using cross-flow fluttering J. Appl. Phys. 109026104. 
[29] Bryant M and Garcia E 2011 Modeling and testing of a novel aeroelastic flutter energy harvester J. Vib. Acoust. 133011010.

[30] Aquino A I, Calautit J K and Hughes B R 2017 Evaluation of the integration of the Wind-Induced Flutter Energy Harvester (WIFEH) into the built environment: Experimental and numerical analysis Appl. Energy 207 61-77.

[31] Wu Y, Li D, Xiang J and Da Ronch A 2017 Piezoaeroelastic energy harvesting based on an airfoil with double plunge degrees of freedom: Modeling and numerical analysis J. Fluid Struct. 74 111-29.

[32] Yan Z, Ragab S A and Hajj M R 2018 Passive control of transonic flutter with a nonlinear energy sink Nonlinear Dynam. 91 577-590.

[33] Zhao L and Yang Y 2018 An impact-based broadband aeroelastic energy harvester for concurrent wind and base vibration energy harvesting Appl. Energy 212 233-243.

[34] Stanton S C, McGehee C C and Mann B P 2009 Reversible hysteresis for broadband magnetopiezoelastic energy harvesting Appl. Phys. Lett. 95174103.

[35] Harne R L and Wang K W 2013 A review of the recent research on vibration energy harvesting via bistable systems Smart Mater. Struct. 22023001.

[36] Stanton S C, McGehee C C and Mann B P 2010 Nonlinear dynamics for broadband energy harvesting: Investigation of a bistable piezoelectric inertial generator Physica D. 239 640-653.

[37] Zhou S, Cao J, Inman D J, Lin J, Liu S and Wang Z 2014 Broadband tristable energy harvester: Modeling and experiment verification Appl. Energy 133 33-39.

[38] Naseer R, Dai H L, Abdelkefi A and Wang L 2017 Piezomagnetoelastic energy harvesting from vortexinduced vibrations using monostable characteristics Appl. Energy 203 142-153.

[39] Zhang L B, Abdelkefi A, Dai H L, Naseer R and Wang L 2017 Design and experimental analysis of broadband energy harvesting from vortex-induced vibrations J. Sound Vib. 408 210-219.

[40] Huynh B H and Tjahjowidodo T 2017 Experimental chaotic quantification in bistable vortex induced vibration systems Mech. Syst. Signal Pr. 85 1005-1019.

[41] Alhadidi A H, Abderrahmane H and Daqaq M F 2016 Exploiting stiffness nonlinearities to improve flow energy capture from the wake of a bluff body Physica D. 337 30-42. 
[42] Zhou S, Cao J, Erturk A and Lin J 2013 Enhanced broadband piezoelectric energy harvesting using rotatable magnets Appl. Phys. Lett.102 173901.

[43] Tiersten H F 2006 On the Derivation of the Equation for the Deflection of Thin Beams Math. Mech. Solids 11 176-180.

[44] Kuethe A M and Chow C Y 1998 Foundations of aerodynamics: Bases of aerodynamic design (New York: Wiley).

[45] Yang T Y and Han A D 1976 Flutter of thermally buckled finite element panels AIAA J. 14 975-957.

[46] Tang D M and Dowell E H 1996 Comments on the ONERA stall aerodynamic model and its impact on aeroelastic stability J. Fluid Struct. 10 353-366.

[47] Rao S S and Yap F F 2011 Mechanical vibrations (Boston: Prentice Hall). 